Draft VERSiOn OCTOBER 15, 2018

Preprint typeset using LATEX style emulateapj v. 2/16/10

\title{
HORIZON RUN 3: TOPOLOGY AS A STANDARD RULER
}

\author{
Robert Speare ${ }^{1}$, J. Richard Gott ${ }^{2}$, Juhan Kim ${ }^{3}$, and Changbom Park ${ }^{4}$ \\ Draft version October 15, 2018
}

\begin{abstract}
We study the Physically Self Bound Cold Dark Matter Halo distribution which we associate with the massive galaxies within the Horizon Run 3 to estimate the accuracy in determination of the cosmological distance scale measured by the topology analysis. We apply the routine "Contour 3D" to 108 Mock Survey of $\pi$ steradians out to redshift $\mathrm{z}=0.6$, which effectively correspond to the SDSSIII BOSS survey, and compare the topology with that of a Gaussian Random Phase Field. We find that given three separate smoothing lengths $\lambda=15,21$, and $34 h^{-1} \mathrm{Mpc}$, the least $\chi^{2}$ fit genus per unit volume g yields a $1.7 \%$ fractional uncertainty in smoothing length and angular diameter distance to $z=0.6$. This is an improvement upon former calibrations of and presents a competitive error estimate with next BAO scale techniques. We also present three dimensional graphics of the Horizon Run 3 spherical mock survey to show a wealth of large-scale structures of the universe that are predicted in surveys like BOSS.
\end{abstract}

Subject headings: large-scale structure of the universe - cosmology: numerical

\section{INTRODUCTION}

The most popular model for the generation of primordial density fluctuations is the inflationary scenarios (Guth 1981; Linde 1982; Albrecht \& Steinhardt 1982; Linde 1983). This model assumes primordial density perturbations of Gaussian random phase and it has been shown that such initial conditions produce a spongelike topology on large scales (Gott, Melott, \& Dickinson 1986, 1987). At such scales, where the power spectrum has not been transformed by nonlinear growth, the topology of structure in the early universe is well preserved, and small deviations from random phase predictions give important information about primordial nongaussianity, biased galaxy formation, and non-linear clustering (Matsubara 1994; Park, et al. 2005a; Park et al. 2012; Park \& Gott 1991; Park et al. 1998).

The genus statistic is central to these studies, and is now a well-tested quantitative measure Gott, Melott, \& Dickinson 1986; Gott. Weinberg, \& Melott 1987; Hamilton. Gott, and Weinberg 1986; Gott et al. 1989; Vogeley et al. 1994; Hikage et al. 2002, 2003; Choi et al. 2010; Park, et al. 2005a; Park et al. 2005b), having been applied to both the SDSS LRG sample (Gott et al. 2009; Strauss et al. 2002; Eisenstein et al. 2011), and the CMB (Park et al. 1998). It's utility lies in the existence of the "genus curve", an analytical expression for genus as a function of density, which allows comparison of observed topology with that expected from a standard big bang inflationary model (Hamilton, Gott, and Weinberg 1986).

So far, fitting the Gaussian random phase (hereafter, GRP) genus curve to mock surveys in a $\Lambda$ CDM cos-

\footnotetext{
${ }^{1}$ New York University Abu Dhabi, PO Box 129188, Abu Dhabi, UAE, robert.speare@nyu.edu

2 Department of Astrophysical Sciences, Peyton Hall, Princeton University, Princeton, NJ 08544-1001, USA

${ }^{3}$ Center for Advanced Computation, Korea Institute for Advanced Study, Heogiro 85, Seoul 130-722, Korea

${ }^{4}$ School of Physics, Korea Institute for Advanced Study, Heogiro 85 , Seoul 130-722, Korea
}

mology has been remarkably successful. The genus has now been suggested as a cosmic standard ruler (Park \& Kim 2010) and a means for probing dark energy (Park \& Kim 2010; Zunckel, Gott, \& Lunnan 2011; Slepian. Gott, \& Zinn 2013). The Baryon Acoustic Oscillation (BAO) feature, detectable in the power spectrum and galaxy two point correlation function, is the established "standard ruler" (Anderson et al. 2012), with a reported fractional uncertainty in angular diameter distance to $z=0.6$ of $1.1 \%$ expected for the SLOAN survey when completed. Now, with the introduction of ever larger galaxy samples, such as the CMASS Data Release 10 sample of the SDSS-III Baryon Oscillation Spectroscopic Survey (BOSS), topology is becoming another attractive technique for probing the expansion of the universe and constraining the equation of state of Dark Energy. We apply the genus to 108 LRG mock surveys, derived from the Horizon Run $3 N$-body Simulations (Kim et al. 2011), in order to ascertain the statistical accuracy of said "topological distance measure".

\section{THE GENUS STATISTIC}

Gott, Melott, \& Dickinson (1986) presented the genus as a reliable description of topology. Traditionally, the genus comes from the Gauss-Bonnet theorem, which states that the integral of gaussian curvature $K=$ $1 /\left(r_{1} r_{2}\right)$ (where $r_{1}$ and $r_{2}$ are the principle radii) over a compact two-dimensional surface is given by

$$
\int K d A=4 \pi\left(1-G_{b}\right) .
$$

We use a slightly altered form of the Gauss-Bonnet genus, $G=G_{b}-1$, so that it has a more intuitive meaning for Cosmology

$$
G=(\# \text { of doughnut holes })-(\text { \# of isolated regions }) .
$$

See Park et al. (2013) for relation to the Euler characteristic and the Betti numbers. With this definition, the genus of a sphere is $G=-1$; a toroid, $G=0$; three isolated spheres, $G=-3$; a figure 8 pretzel, $G=1$ 
(two holes, one isolated body). Essentially, the genus is a measure of connectivity. A highly connected structure - such as a sponge - will have many holes, a single body, and therefore a large, positive genus. A sparse array of objects - a meatball topology (Soneira \& Peebles 1978; Press \& Schechter 1974) - will have many isolated regions, relatively few holes, and therefore a negative genus. An array of isolated voids will also produce a negative genus.

To calculate the genus we smooth the Horizon Run 3 Physically Self Bound Subhalo distribution Kim et al. (2011) with a Gaussian smoothing ball of radius $\lambda$ (Eq. 8).

We picked the most massive physically bound subhalos to match the number density of LRG galaxies projected for the SLOAN III survey when completed. The Horizon Run 3 is a Cold Dark Matter simulation. We make the simple assumption that the most luminous red galaxies will from in the centers of the most massive cold dark matter halos. In the simulation, the most massive subhalos (> 30 CDM particles) are identified that physically bond and not tidally disruptable by larger structures these we associate with LRG galaxies.

We then create iso-density contour surfaces of the smoothed density distribution, labeling them by $\nu$, which is related to the volume fraction $f$ on the high density side of the contour by

$$
f=\frac{1}{\sqrt{2 \pi}} \int_{\nu}^{\infty} e^{-x^{2} / 2} d x,
$$

Where $x$ is the density parameter. The value $f=50 \%$ corresponds to the median volume fraction contour $(\nu=$ 0 ). For GRP initial conditions the genus curve is

$$
g_{r f}(\nu)=A\left(1-\nu^{2}\right) e^{-x^{2} / 2} .
$$

Where the amplitude $A=\left(1 / 2 \pi^{2}\right)\left(\left\langle k^{2}\right\rangle / 3\right)^{3 / 2}$, and $\left\langle k^{2}\right\rangle$ is the average value of the squared wave vector, $k^{2}$ in the smoothed power spectrum (Gott, Melott, \& Dickinson 1986); or, the slope of the two-point correlation function.

The shape of a genus curve, and its deviation from the random phase prediction, can be parametrized by several variables. First, there is the $\chi^{2}$ best fit amplitude, which is measured by fitting the GRP curve ( Eq. 4) to the observed curve. This gives information about the power spectrum and phase correlation of the density fluctuation. Secondly, there are three variables which characterize deviations from a GRF (Park, Gott, \& da Costa 1992):

$$
\begin{aligned}
\Delta \nu & =\frac{\int_{-1}^{1} g(\nu) \nu d \nu}{\int_{-1}^{1} g_{\mathrm{rf}}(\nu) \nu d \nu}, \\
A_{V} & =\frac{\int_{-2.2}^{-1.2} g(\nu) d \nu}{\int_{-2.2}^{-1.2} g_{\mathrm{rf}}(\nu) \nu d \nu}, \\
A_{C} & =\frac{\int_{1.2}^{2.2} g(\nu) d \nu}{\int_{1.2}^{2.2} g_{\mathrm{rf}}(\nu) \nu d \nu},
\end{aligned}
$$

where $g_{\mathrm{rf}}$ is the best-fit random phase curve (Eq. 4). $\Delta \nu$ measures any shift in the central part of the genus curve. The GRP curve has $\Delta \nu=0$. A negative value of $\Delta \nu$ is called a meatball shift, caused by a greater prominence of isolated high-density structures, pushing the genus curve to the left. $A_{V}$ and $A_{C}$ measure the relative number of voids and clusters with respect to GRP expectations.

\section{THE $N$-BODY SIMULATIONS}

The Horizon Runs, provided by the Korean Institute of Advanced Study (KIAS), provide some of the best raw material for calibrating topological study of LRG surveys (Park, et al. 2005a; Kim et al. 2011). These $N$ body simulations replicate the topology of the SDSS LRG's exquisitely (Gott et al. 2009; Eisenstein, et al. 2001). We use the Horizon Run 3 (HR3) dataset exclusively, which adopts a pressureless cold dark matter cosmology with a pure cosmological constant $w_{\Lambda}=-1$. The basic HR3 cosmological parameters were fixed by the WMAP5 data (Spergel et al. 2003; Komatsu et al. 2011; Hinshaw et al. 2013) and the initial linear power spectrum was calculated with the CAMB source code (Lewis \& Bridle 2002). The entire simulation is a cube of 374 billion particles, spanning a volume of $\left(10.815 h^{-1} \mathrm{Gpc}\right)^{3} 5$ Initial redshift was $z=27$ and $N_{\text {step }}=600$ discrete timesteps were taken.

\subsection{Mock LRG Survey Construction}

The selection of cold dark matter halos uses the Friend of Friend algorithm, where separation cut off distance is $20 \%$ of the mean separation distance. To improve cluster identification, HR3 searches for Physically Self Bound (PSB) subhalos that are gravitationally self-bound and not tidally disruptable (Kim \& Park 2006). This provides a substantial increase in the similiarity between simulation and observational data, as these dark matter subhalos are sites for LRG formation.

To simulate the SDSS survey dimensions, HR3 places 27 observers evenly within its cubical volume and allows each observer to see out to a redshift of $z<0.7$. This crates 27 independent, non-overlapping spherical regions. The co-moving positions and velocities of all CDM particles are saved as they cross their past light cone and PSB subhalos are identified from this data. In preparation for the SDSS-III LRG catalogue, it was assumed that a volume-limited sample would yield a constant number density of $3 \times 10^{-4}\left(h^{-1} \mathrm{Mpc}\right)^{3}$. In order to match this prediction, the minimum mass limit of the PSB subhalos was varied with redshift and the absolute minimums were set to $9.75 \times 10^{12} h^{-1} \mathrm{M}_{\odot}$. Given these parameters, the physical properties of the HR3 mock surveys match very well with the most recent LRG surveys (Choi et al. 2010; Gott et al. 2009, 2008).

\section{METHODS}

\subsection{Smoothing and Discretization}

We smooth the 27 past lightcone PSB subhalo distributions with a gaussian smoothing ball

$$
W(\vec{r})=\frac{1}{(2 \pi)^{3 / 2}} e^{-\frac{r^{2}}{2 \lambda^{2}}},
$$

smearing structure on scales smaller than $\lambda$. The Mock survey data is placed into a three-dimensional pixel grid

5 For comparison, this volume is 8800 times larger than the Millenium Run (Springel et al. 2005). 


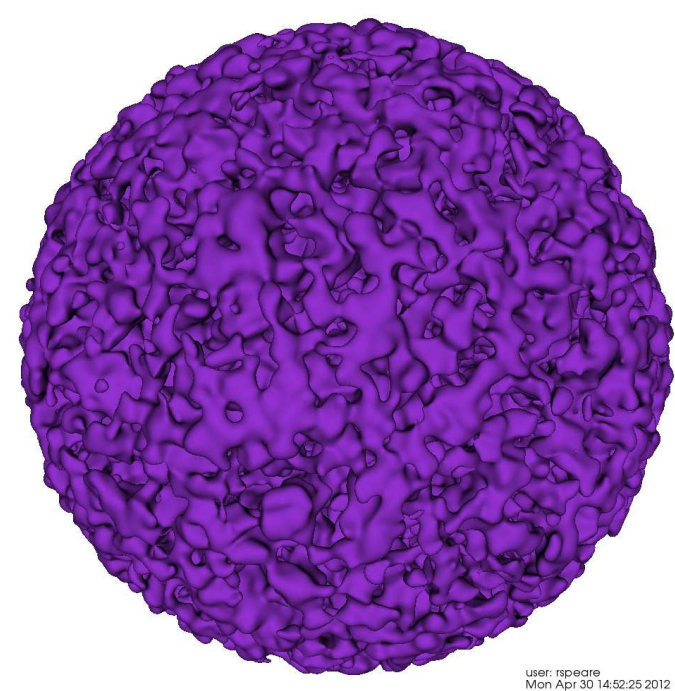

FIG. 1.- A spherical Horizon Run 3 Mock survey out to redshift $z=0.7$. The PSB subhalo counts have been smoothed with a Gaussian smoothing ball of $\lambda=34 h^{-1} \mathrm{Mpc}$. See $\mathrm{A}$ for 3D plots of the Horizon 3 data.

of density values, and we choose $\lambda$ to always be greater than 2.5 pixel sidelengths $s$. For cold dark matter models, smoothing with a Gaussian recovers the topology of the initial density field, provided that the smoothing length $\lambda$ is sufficiently greater than the correlation length $R_{0}$ and non-linear effects are avoided 6 .

\subsection{Conversion and Trimming}

With this smoothed Mock Survey in hand, we convert from co-moving spherical coordinates to redshift coordinates, using a comoving line of sight distance formula (Hogg 1999). PSB subhalo peculiar velocities are converted into redshift distortions by

$$
\Delta z=\frac{\overrightarrow{v_{\mathrm{r}}}}{c}=\frac{\hat{r} \cdot \vec{v}_{\mathrm{pec}}}{c},
$$

where $\vec{v}_{r}$ is the radial velocity, $\hat{r}$ is the unit radial vector, and $\vec{v}_{\text {pec }}$ is the cartesian peculiar velocity of the subhalo. After redshift converting and correcting, we save PSB subhalo counts within a grid of dimensions $650^{3}$, with cubical pixel volume of $s^{3}=\left(6 h^{-1} \mathrm{Mpc}\right)^{3}$. The entire grid spans a volume of $\left(1950 h^{-1} \mathrm{Mpc}\right)^{3}$.

We then apply an angular mask, splitting the 27 perfectly spherical mock surveys into four quadrants each of $\pi$ steradians and radius $z=0.6$, to approximate the area of sky coverage and depth in the SLOAN III survey. With these $4 \times 27=108$ smoothed mock surveys in hand, we calculate the genus using a polygonal approximation scheme developed by Weinberg (1988); Hamilton, Gott, and Weinberg (1986) called "Contour 3D", which adds up angle deficits at pixel vertices.

\section{USING TOPOLOGY AS A STANDARD RULER}

An application of quantitative topology being applied to the SDSS LRG sample - other than testing the gaussianity of initial density fluctuations - is to measure cosmological parameters, such as those governing the expansion history of the universe. This can be done by

${ }^{6} R_{0}$ is approximately $5 h^{-1} \mathrm{Mpc}$ for LRG measuring the genus statistic within a fixed volume at different redshifts. In the instance of $N$-body simulations, one knows the correct cosmological model and therefore the correct transformation $r \rightarrow z$. One smooths the density field with a known smoothing length $\lambda$ and then measures the median density genus within a volume $V$. This yields $g=G / V$, genus per unit volume, which one can use to indirectly measure any physical volume by counting structures. In order to more explicitly state the smoothing length dependence, the dimensionless quantity $g \lambda^{3}$ is often used, which is simply the genus per cubic smoothing length. This quantity can be analytically calculated from a full set of cosmological parameters and a linear power spectrum. Such a function $g \lambda^{3}(\lambda)$ has been examined closely for the WMAP3 and WMAP5 parameters (see fig. 1 of Park \& Kim 2010 and fig. 1 of Zunckel, Gott, \& Lunnan 2011, drawn by Y.R. Kim.)(see Fig. 2).

In practice, we do not know the true cosmological model. Let us illustrate the effects of applying an incorrect cosmological model to a survey sample. If we underestimate the expansion rate of the universe $H_{0}$, then our conversion from redshift to comoving space will put celestial objects too far from the Earth. This causes an overestimation of survey volume. For a homogeneous and isotropic survey, genus is linearly proportional to volume and therefore an overestimation of $V$ will drive the genus at a certain smoothing length up $(G(\lambda) \uparrow)$. At the same time however, we have also adopted a comoving smoothing length $\lambda$ that is larger than intended. This will change the actual scale of study and erase all structure beneath scale by convolution, decreasing the genus $(G(\lambda) \downarrow)$. Luckily, the net effect is detectable since the amplitude $G$ of the genus curve effectively measures the slope of the power spectrum at the scale $\lambda$, which is not scale invariant (Park \& Kim 2010).

Our procedure for measuring angular diameter comoving distance to $z=0.6$ is straightforward. We assume a $\Lambda$ CDM flat cosmological model. $\Omega_{m}, h$, and $\Omega_{\Lambda}$ come from CMB fits with $l>210$ which are insensitive to $w_{\Lambda}$ because dark energy has negligible influence at recombination. These values are used to construct the power spectrum and from that, $g \lambda^{3}(\lambda)$ (see Fig. 2). Now we measure $g \lambda^{3}$ and get a value; we look on our analytical plot - Fig. 2 - and find the true value of $\lambda$, which we will call $\lambda_{\text {true. }}$. If this is $1 \%$ smaller than the initial value of $\lambda$ that one used, it means that the co-moving distance out to $z=0.6$ is also $1 \%$ smaller than previously thought. In this way one can measure co-moving distance out to $z=0.6$. And, with this as one data point one can fit a cosmological model, leaving $w_{\Lambda}$ as a parameter (Park \& Kim 2010).

If the intial cosmological model is slightly wrong (i.e. $w_{\Lambda}$ may not be exactly -1 , or may vary with time; Slepian. Gott, \& Zinn 2013), this is inconsequential because we are just measuring the topology - counting the total number of structures inside $z=0.6$. If the radial co-moving distance inside this volume is proportionately in error it will make no difference, as that will just distort shapes and structures slightly without altering their count (see Zunckel. Gott. \& Lunnan 2011). An rms cosmic variance in the total genus $\sigma_{g}$ out to $z=0.6$ in a survey sample will cause a fractional $\mathrm{rms}$ error of $\sigma_{g} / g$ in 


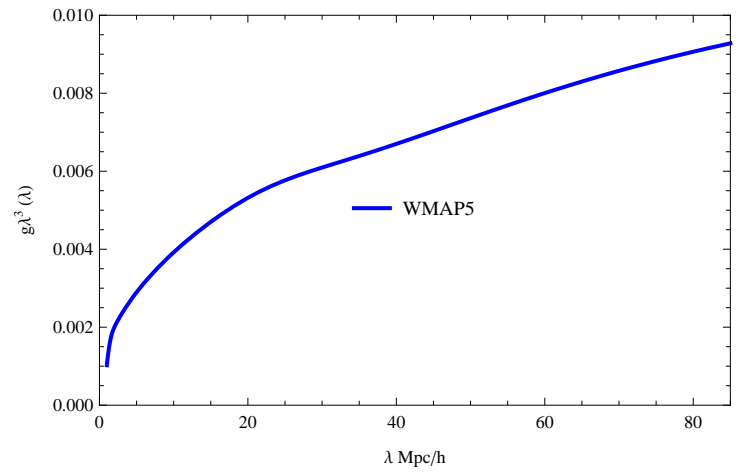

FIG. 2.- Genus per cubic smoothing length $g \lambda^{3}$ for the WMAP5 parameters $\left(\Omega_{m}=0.26, H_{0}=74\right)$, assuming a flat $\Lambda$ CDM cosmology (taken from Fig. 1 of Zunckel, Gott, \& Lunnan 2011, calculated by Young Rae Kim).

$g \lambda^{3}$; and given the slope of the curve, $\left(g \lambda^{3}\right)^{\prime}$ at the applied $\lambda$, this will introduce an rms error in $\lambda$ and therefore in co-moving distance at $z=0.6$ of:

$$
\left(g \lambda^{3}\right)^{\prime} \frac{\sigma_{\lambda}}{\lambda}=\frac{\sigma_{g}}{g}
$$

\subsection{Uncertainties in such a ruler}

We examine the statistical variance of genus per unit volume $g$ in the Horizon Run 3 mock surveys, which is far from an "ideal" measurement.

An "ideal" measurement of $g$ would be examining the initial density field in comoving space. If the initial conditions were of GRP, one would expect excellent agreement between the observed genus curve and the theoretical GRP curve; however, finite sample size, even at this level, introduces an error because of no power at large scales (or, larger than the simulation box size). The next best measurement of $g$ would be examining the final conditions of the entire $N$-body simulation in comoving space, which erases a portion of the cosmic variance associated with small survey size, but is subject to the effects of non-linear gravitational infall and galaxy formation bias. An unavoidable source of error, "ideal" or otherwise, is finite pixel resolution, which applies a smoothing scale to the data and destroys structure smaller than pixel size $s$.

Observation of $g$ in comoving space has obvious advantages to observation in redshift space, since one has complete knowledge of all PSB subhalo positions and velocities. It has been found that the redshift correction for peculiar velocity presents the worst source of error for the $\chi^{2}$ best fit amplitude of the genus curve (Choi et al. 2010). The application of peculiar velocity redshift corrections is in essence a smoothing routine of its own, in that real-space structures are radially smeared due to "fingers of god" effects. This effectually raises the observed smoothing parameter $\lambda$ slightly and yields a lower $\chi^{2}$ best fit genus amplitude. The choice of survey volume, specifically volume to surface area ratio, also creates error because of data being "smoothed out" of the survey region. The complicated boundaries of the SDSS present a cause for concern; particularly the three thin stripes along the southern Galactic cap, which are ignored altogether during genus analysis.

An SDSS measurement of $g$ uses a finite, redshift space sample, where the aforementioned sources of error apply: the cosmic variance associated with small survey size; non-linear clustering; boundary effects; and redshift space distortion. The situation sounds daunting, but because of its size, the Horizon Run 3 provides an ensemble of tests. We split the 27 HR3 spherical mock surveys into four quadrants, thereby acquiring 108 "genus experiments" for a chosen smoothing length $\lambda$. Gott et al. (2009) have reported the genus amplitude of the SDSS LRG to within $5 \%$ accuracy. Based upon our results (see Table 1), we believe that this fractional uncertainty can be reduced to of $1 \%$.

\section{RESULTS}

We measured the genus per cubic smoothing length for $\lambda=15,21$, and $34 h^{-1} \mathrm{Mpc}$, studying the random and systematic error over 108 HR3 mock surveys. For $\lambda=15 h^{-1} \mathrm{Mpc}$, the fractional uncerainty in genus per cubic smoothing length was less than one percent, which translates to a fractional uncerainty in smoothing length - and angular diameter distance - of approximately $2.1 \%$ (Table 11).

Treating the variance at $\lambda=15,21$, and $34 h^{-1} \mathrm{Mpc}$ as statistically independent - since HR3 adopts a random phase model and the smoothing volumes are significantly different - we add the three smoothing length rms errors in quadrature

$$
\frac{1}{\sigma_{\mathrm{eq}}^{2}}=\frac{1}{\sigma_{1}^{2}}+\frac{1}{\sigma_{2}^{2}}+\frac{1}{\sigma_{3}^{2}},
$$

yielding a $1.69 \%$ fractional uncertainty in smoothing length and angular diameter distance out to $z=0.6$. Combining only the 21 and $34 h^{-1} \mathrm{Mpc}$ samples, we get a $2.97 \%$ fractional uncertainty in smoothing length.

With 108 samples in hand, our fractional "uncertainty of the uncertainty" is $1 / \sqrt{2(N-1)}=6.8 \%$. It is notable that the systematic effect for the $21 h^{-1} \mathrm{Mpc}$ sample was very small, $-0.84 \%$, and that the $\chi^{2}$ best-fit genus amplitudes modeled the $g \lambda^{3}$ curve extraordinarily well (Fig. 3).

\section{DISCUSSION}

With these results in hand, it is important to continue refining topological study of the SDSS LRG sample with $N$-body simulations. Extremely large cubes like HR3 allow for tight description of the cosmic variance in genus per unit volume $g$ and smoothing length $\lambda$. This statistical knowledge translates directly to the measurement of cosmological parameters such as $w$. A possible extension of this work is to more accurately model the SDSS survey with 108 less "ideal" masks. Another possible extension is to measure the variance in genus per cubic smoothing length $g \lambda^{3}$ for a large number of $\lambda$ 's, perhaps iterating from $15 h^{-1} \mathrm{Mpc}$ to $34 h^{-1} \mathrm{Mpc}$ in small increments $\Delta \lambda<0.2 h^{-1} \mathrm{Mpc}$. Smooth plots of $\sigma_{g \lambda^{3}}$ and $\lambda-\lambda_{\text {true }}$ as a function of $\lambda$ could yield useful information about the evolution of random and systematic error with scale.

We thank the Princeton Department of Astrophysical Sciences, Princeton NJ, where this work was completed. We thank the support of the Supercomputing Center/Korea Institute of Science and Technology Infor- 


\begin{tabular}{|l|l|l|l|}
\hline & $\lambda=15 \mathrm{Mpc} / h$ & $\lambda=21 \mathrm{Mpc} / h$ & $\lambda=34 \mathrm{Mpc} / h$ \\
\hline$g \lambda^{3} \times 10^{3}$ & 4.762 & 5.403 & 6.271 \\
$\sigma_{g \lambda^{3}} \times 10^{3}$ & 0.04380 & 0.6732 & 1.358 \\
$\frac{\sigma_{g \lambda^{3}}}{g \lambda^{3}}$ & $.919 \%$ & $1.245 \%$ & $2.166 \%$ \\
$\lambda_{\mathrm{t}}$ & 15.448 & 20.823 & 32.993 \\
$\frac{\lambda_{t}-\lambda}{\lambda}$ & $2.99 \%$ & $-0.84 \%$ & $-2.96 \%$ \\
$\frac{\sigma_{\lambda}}{\lambda}$ & $2.096 \%$ & $3.215 \%$ & $6.742 \%$ \\
\hline
\end{tabular}

TABLE 1

$g \lambda^{3}$ IS THE AVERAGed $\chi^{2}$ BeST-Fit GENUS PER CUBIC SMOOTHING LENGTh, FOR ALL 108 MOCK SURVEYS - MULTIPLIED BY $10^{3}$ FOR THE READER'S SAKE. $\lambda_{\mathrm{t}}$ IS THE CORRESPONDING "TRUE" SMOOTHING LENGTH FOR THE OBSERVED GENUS PER CUBIC SMOOTHING LENGTH, AS DisCUSSED IN SECTION $5 \sigma_{g \lambda^{3}}$ AND $\sigma_{\lambda}$ REPRESENT THE VARIANCE IN SMOOTHING GENUS PER CUBIC SMOOTHING LENGTH AND SMOOTHING LENGTH. $\left(\lambda_{t}-\lambda\right) / \lambda$ IS THE FRACTIONAL, SYSTEMATIC ERROR IN SMOOTHING LENGTH.

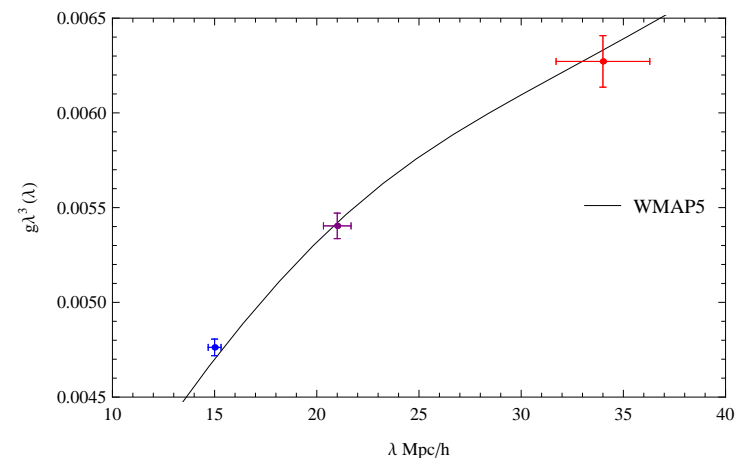

mation with supercomputing resources, including technical support (KSC2011G202) for Horizon Run 3. We also thank Korea Institute for Advanced Study for providing computing resources (KIAS Center for Advanced Computation Linux Cluster System).

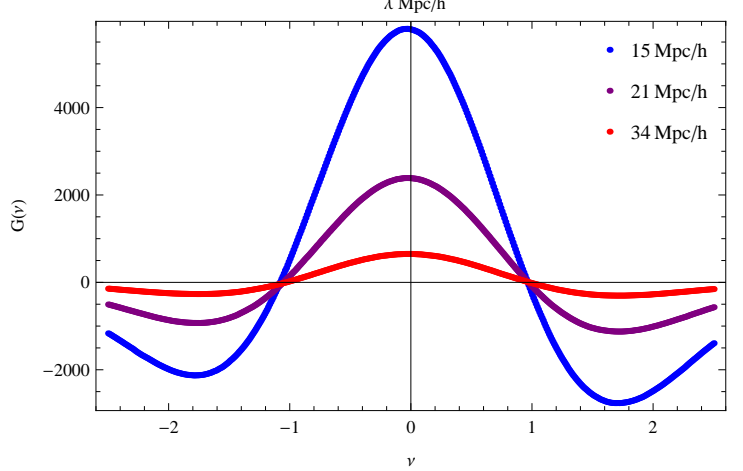

FIG. 3.- Above: genus per cubic smoothing length $g \lambda^{3}$ for the WMAP 5 parameters, with $\chi^{2}$ best-fit data points and $1 \sigma$ error bars. Below: the ensemble averaged genus curves $G(\nu)$ for $\lambda=15$, 21 , and $34 h^{-1} \mathrm{Mpc}$

\section{APPENDIX}

3D GRAPHICS OF THE HORIZON RUN 3

\section{REFERENCES}

Albrecht H. \& Steinhardt P. J. 1982, Phys. Rev. Lett. 48, 1220

Anderson, et al. 2012, MNRAS, 427, 3435

Choi, Y.-Y, Park, C., Kim, J., Gott, J.R., Weinberg, D.H., Vogeley, M.S., Kim, S.S. 2010, ApJS, 190, 181

Eisenstein, D.J., Annis, J., Gunn, J.E., et al. 2001, AJ, 122, 2267

Eisenstein, D.J., Weinberg, D.H., et al. 2011, AJ, 142, 72

Gott, J. R., Melott, A. L., \& Dickinson, M. 1986, ApJ, 306, 341

Gott, J. R., Weinberg, D.N., \& Melott, A.L. 1987, ApJ, 319, 1

Gott, J.R., et al. 1989, ApJ, 340, 625

Gott, J. R., et al. 2008, ApJ, 675, 16

Gott, J.R., et al. 2009, ApJ, 695, L45

Guth, A.H. 1981, Phys. Rev. D, 23, 347

Hamilton, A. J. S., Gott, J. R., \& Weinberg, D. W. 1986, ApJ, 309,1

Hikage, C., et al. (The SDSS Collaboration) 2002, PASJ, 54, 707

Hikage, C., et al. 2003, PASJ, 55, 911
Hinshaw, G., Larson, D., Komatsu, E., et al. 2013, arXiv1212.5226

Hogg, D. W. 1999 arXiv:astro-ph/9905116

Kim, J., \& Park, C. 2006, ApJ, 639, 600

Kim, J., Park, C., Ross, G., Lee, S.M., \& Gott, J.R. 2011, JKAS, 44. 217

Komatsu, E., Smith, K.M., \& Dunkley, J., et al. 2011, ApJS, 192, 18

Linde, A.D. 1982, Phys. Lett. B, 108, 389

Linde, A.D. 1983, Phys. Lett. B, 129, 177

Lewis, A., Bridle, S., 2002, Phys. Rev., D66 103511

Matsubara, T. 1994, ApJ, 434, L43

Park, C., \& Gott, J.R. 1991, ApJ, 378, 457

Park, C., Gott, J.R., \& da Costa, L.N. 1992, ApJ, 392, L51]

Park, C., Colley, W.N., Gott, J.R., Ratra, B., Spergel, D.N., \&

Sugiyama, N. 1998, ApJ, 506, 473 

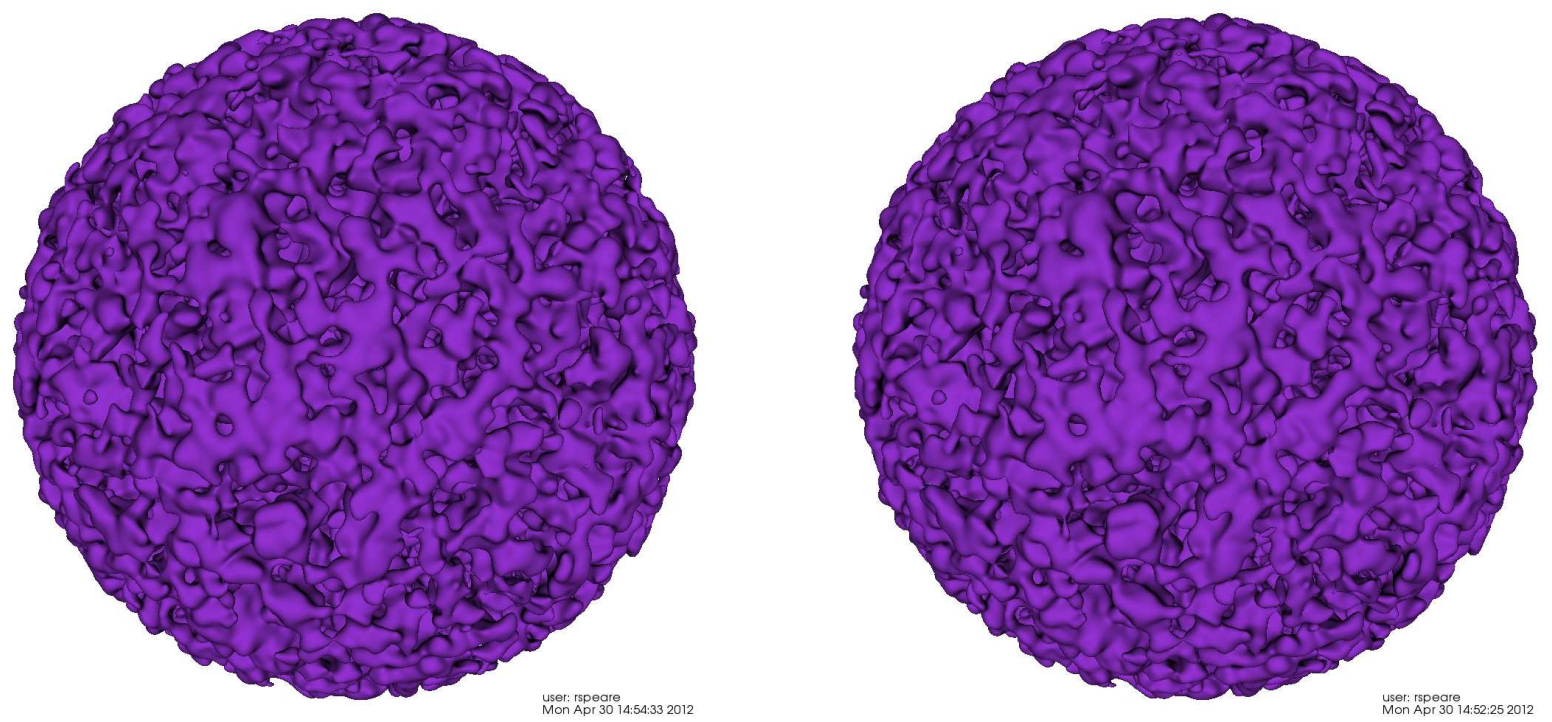

FIG. 4. - Stereo View of the Horizon Run 3 spherical mock survey out to redshift $z=0.7$. In order to see this figure in 3D, cross you eyes until you see three purple sponges. Fuse the middle sponge and bring it into focus. The middle sponge should be in $3 \mathrm{D}$.

Park, C., Kim, J., \& Gott, J.R. 2005a, ApJ, 633, 1

Park, C., et al. (The SDSS Collaboration) 2005b, ApJ, 633, 11

Park, C., \& Kim, Y.-R. 2010, ApJ, 715, L185

Park, C., Choi, Y.-Y., Kim, J., Gott, J.R., Kim, S.S., \& Kim, K.-S. 2012, ApJ, 759, L7

Park, C., Pranav, P., Chingangbam, P., van de Weygaert, R., Jones, B., Vegter, G., Kim, I., Hidding, J. \& Hellwing, W.A. 2013, JKAS, 46, 125

Press, W.H., \& Schechter, P. 1974, ApJ, 187, 425
Slepian, Z., Gott, J.R., \& Zinn, J. 2013, arXiv1301.4611 Soneira, R.M. and Peebles, P. J. E. 1978, A.J., 83, 845 Spergel, D.N., et al. 2003, ApJS, 148, 175

Springel, V. 2005, Nature, 435, 629

Strauss, M.A., et al. 2002, AJ, 124, 1810

Vogeley, M.S., Park, C., Geller, M.J., Huchra, J.P., \& Gott, J.R. 1994, ApJ, 420, 525

Weinberg, D. H. 1988, PASP, 100, 1373

Zunckel, C., Gott, J.R., \& Lunnan, R. 2011, MNRAS, 412, 1401 


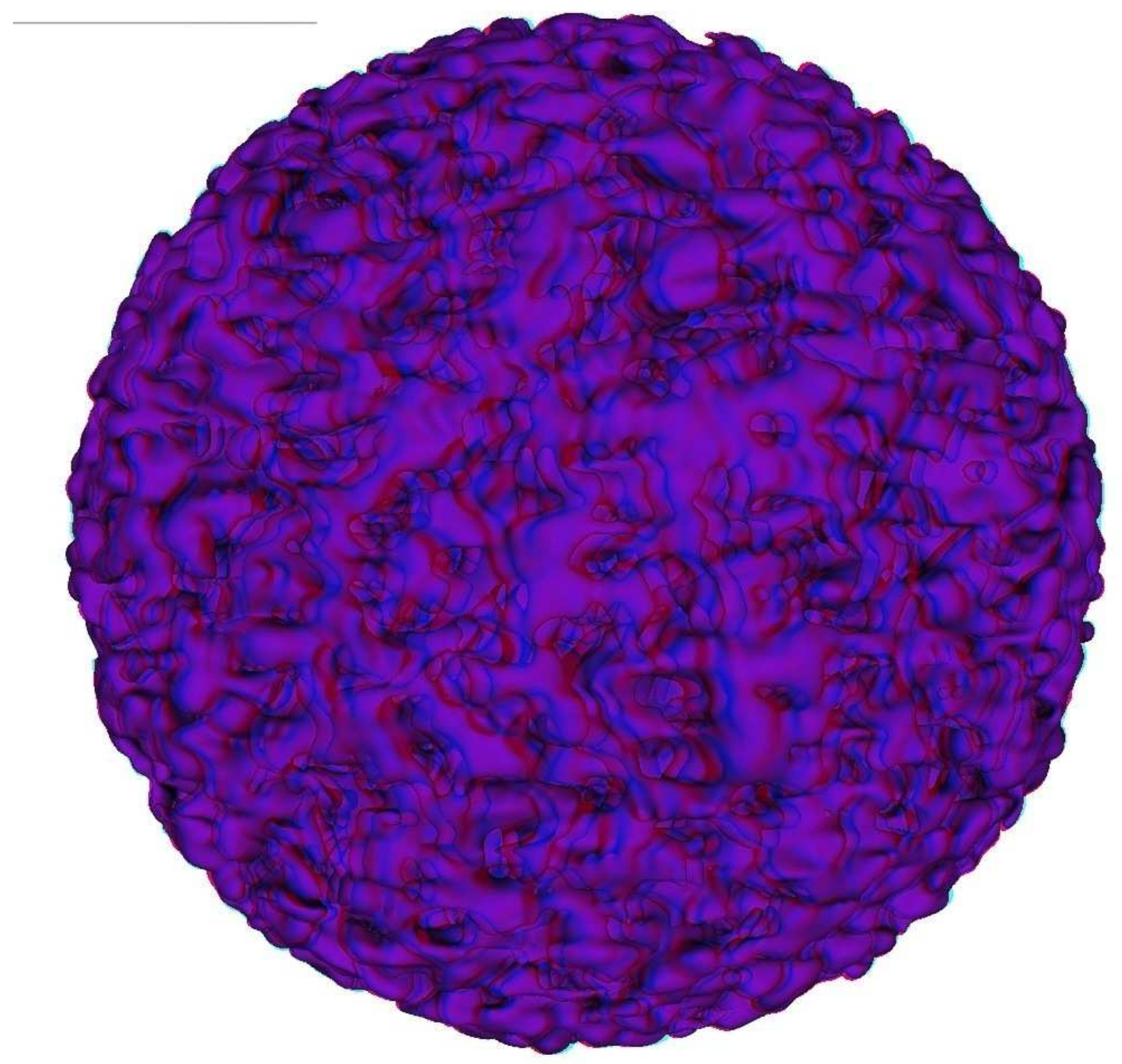

FIG. 5.- A red blue anaglyph of a spherical Horizon Run 3 Mock survey out to redshift $z=0.7$. The PSB subhalo counts have been smoothed with a Gaussian smoothing ball of $\lambda=34 h^{-1} \mathrm{Mpc}$. Requires red-cyan lensed glasses to view. 